\title{
Routes of hysterectomy in women with benign uterine disease in the Vancouver Coastal Health and Providence Health Care regions: a retrospective cohort analysis
}

\author{
Innie Chen MD, Sarka Lisonkova MD PhD, Catherine Allaire MD, Christina Williams MD, \\ Paul Yong MD PhD, K.S. Joseph MD PhD
}

Abstract

Background: Minimally invasive hysterectomies performed vaginally or laparoscopically are associated with decreased perioperative morbidity. We examined temporal trends and patient and hospital factors associated with the routes of hysterectomy used in the Vancouver Coastal Health and Providence Health Care regions in British Columbia.

Methods: We performed a retrospective cohort study of all women who had an elective hysterectomy for a benign indication between 2007 and 2011 in 8 hospitals in the region. Logistic regression modeling with mixed effects was used to estimate adjusted odds ratios and $95 \%$ confidence intervals for patient and hospital characteristics associated with the route of hysterectomy.

Results: The study involved 4372 women who underwent abdominal (52.3\%), vaginal (25.5\%) or laparoscopic (22.3\%) hysterectomy. From 2007 to 2011, the number of abdominal hysterectomies performed decreased from $58.4 \%$ to $47.7 \%$, the number of vaginal hysterectomies performed decreased from $27.5 \%$ to $21.1 \%$ and the number of laparoscopic hysterectomies performed increased from $14.2 \%$ to $31.2 \%$ ( $p<0.001$ for all trends). Patient factors associated with laparoscopic versus abdominal hysterectomy included young age, pain or prolapse indication, absence of fibroid indication, absence of concurrent gynecologic procedure, rural residence and lower socioeconomic status. Patient factors associated with vaginal hysterectomy included older age, prolapse indication and concurrent procedure for prolapse. Hospital location and size were not significantly associated with vaginal hysterectomy, but urban hospital location was associated with laparoscopic hysterectomy.

Interpretation: The proportion of minimally invasive hysterectomies is increasing and represents approximately half of all hysterectomies performed in the Vancouver Coastal Health and Providence Health Care regions. Vaginal hysterectomies are associated with patient characteristics, whereas laparoscopic hysterectomies are associated with patient and hospital characteristics.

H ysterectomy is the most frequently performed gynecologic operation: approximately 50000 are performed each year in Canada. ${ }^{1,2}$ Traditionally, hysterectomies have been performed via the abdominal route, which involves a relatively large abdominal incision. However, hysterectomies performed via the vaginal route or using laparoscopy (via smaller incisions) have been shown to be associated with substantially faster recovery and decreased operative morbidity. ${ }^{3-5}$ The evidence in favour of minimally invasive hysterectomies is reflected in the current national guidelines. ${ }^{6,7}$ Vaginal hysterectomy is the preferred type of minimally invasive hysterectomy, but its use may be limited in patients with concurrent pelvic disease, a large uterus or in patients lacking uterine descent. For these patients, the laparoscopic approach may avoid a prolonged recovery period and increased morbidity. ${ }^{2,8}$ The concept of technicity, i.e., the use of a vaginal or laparoscopic approach, was introduced to measure the proportion of minimally invasive hysterectomies among all hysterectomies, and it has been proposed as a quality indicator in gynecologic practice., ${ }^{2,9}$

Despite the wealth of evidence favouring the minimally invasive approach for benign gynecologic disease, a national study of hysterectomies performed from 1981 to 1997 showed that most hysterectomies performed in Canada were abdominal, ${ }^{1}$ and a recent national survey suggested that nearly $40 \%$ of gynecologists were not offering hysterectomy by laparoscopy. ${ }^{10}$ More recent reports from Ontario ${ }^{11,12}$ and Quebec ${ }^{13}$ suggested that $40 \%-45 \%$ of hysterectomies are performed

Competing interests: None declared.

This article has been peer reviewed.

Correspondence to: Innie Chen, ichen@toh.on.ca

CMAJ Open 2013.DOI:10.9778/cmajo.20130080 
with minimally invasive routes. However, these studies were limited by the absence of adequate information on patient and clinical factors.

We conducted a study that examined the routes used for hysterectomy in the Vancouver Coastal Health and Providence Health Care regions of British Columbia. Our primary objective was to study temporal trends in hysterectomy practice. Our secondary objective was to identify patient and hospital factors associated with the different routes of hysterectomy. In addition, we compared surgical time, length of hospital stay, readmission to hospital and rates of emergency visits for each type of hysterectomy. We hypothesized that the proportion of minimally invasive hysterectomies increased over time. Because patient choice and the surgeon performing the procedure may influence the route used for the hysterectomy, we also hypothesized that, in addition to clinical factors such as the presence of fibroids and endometriosis, patient sociodemographic factors, and hospital and geographic settings would also be associated with the route of hysterectomy chosen.

\section{Methods}

\section{Setting}

The Vancouver Coastal Health and Providence Health Care regions cover a wide urban and rural geographic area that includes the city of Vancouver, Vancouver's North Shore, Richmond, the Sea-to-Sky Highway, Sunshine Coast, Bella Bella, Bella Coola, the Central Coast and the surrounding areas. Together, they serve more than one million people, which is over a quarter of the provincial population.

\section{Design}

We used a population-based retrospective cohort design that included women who had an elective hysterectomy for benign gynecologic indications between April 1, 2007, and December 31, 2011. Women who were not residents of British Columbia at the time of surgery were excluded.

\section{Data sources}

Data from the Discharge Abstract Database were linked to the Operating Room Management Information System database and the Emergency Department Surveillance System. Discharge Abstract Database is a national hospital administrative database that captures administrative, clinical and demographic data for patients who are discharged from hospital. The database uses consistent data collection procedures that undergo routine validation and quality control. ${ }^{14}$ The use of this system for studying the route used for hysterectomy was reported previously, ${ }^{11,12}$ and the majority of the information used for this study was taken from this data source. The Operating Room Management Information System is a database system dedicated to the capture of detailed clinical and resource utilization information pertaining to each surgical episode. ${ }^{15}$ The dataset was used to obtain additional information regarding the surgical cases and the operative times. The Emergency Department Sur- veillance System was developed by the Vancouver Coastal Health Authority to capture visits to an emergency department in 9 acute care hospitals across the region for the purpose of population surveillance. The data are summarized, reviewed and validated against charts at regular intervals, ${ }^{16}$ and this information was used to determine whether a visit to the emergency department occurred in the observation period for each patient. Together, these 3 data sources captured demographic, hospitalization, surgical and emergency department information.

\section{Statistical analysis}

Procedure codes of the Canadian Classification of Health Interventions (CCI) (version 10) were used to classify hysterectomies into abdominal (CCI 1RM89LA), vaginal (CCI 1RM89CA) and laparoscopic (CCI 1RM89AA and 1RM89DA). Cases of partial excision of the uterus (vaginal hysterectomy, CCI 1RM87BAGX and 1RM87CAGX; laparoscopic hysterectomy, 1RM87DAGX; and for abdominal hysterectomy, 1RM87LAGX) were identified as subtotal hysterectomies using the Operating Room Management Information System database. Minimally invasive hysterectomy was defined as vaginal or laparoscopic hysterectomy.

Rural residences were defined by residential postal codes corresponding to areas with $<10000$ inhabitants. ${ }^{17}$ Socioeconomic status was inferred from residential postal codes using neighbourhood income quintiles (from lowest (1) to highest (5) income level) relative to the income distribution in British Columbia in 2006, which is considered an adequate approximation of household income. ${ }^{18}$ The International Classification of Diseases diagnostic codes (ICD-10) were used to categorize the indication for hysterectomy: fibroids (ICD-10 D25), menstrual bleeding disorders (N92 and N93), endometriosis (N80), pain (N94), prolapse (N81) and other. Concurrent gynecological procedures were identified from procedure codes in the Discharge Abstract Database and included ovarian procedures (CCI $1 \mathrm{RB}$ and 1RD89), prolapse (1RS51, 1RS74, 1RS80, 1PL74, 1PL80, $1 \mathrm{NQ74}$ and 1NQ80), and other. Indications and procedures not corresponding to these categories were categorized as other. Hospital factors included the size of the hospital ( $<100$ beds v. $\geq 100$ beds) and whether the hospital was in an urban or rural setting, according to the postal code of the hospital. Information related to surgical outcomes, such as operative time, length of hospital stay, return visit to the emergency department and readmission to hospital within 60 days after discharge was also collected.

Baseline patient and hospital characteristics and surgical outcomes were compared between women who had vaginal, laparoscopic and combined minimally invasive hysterectomies and women who had abdominal hysterectomies. Continuous variables were compared using a t test or Wilcoxon rank sum test to assess the statistical significance of differences, whereas categorical variables were compared using a $\chi 2$ or Fisher exact test. The Cochran-Armitage test for trend was used to assess the statistical significance of temporal trends for the proportion of each type of hysterectomy. We used mixed-effects 
models with a logit link function (GLIMMIX procedure) to identify independent predictors for different routes of hysterectomy. The results were expressed as adjusted odds ratios (adjusted OR) and 95\% confidence intervals (95\% CI). In the multivariate regression analysis, only the main indication for hysterectomy was considered as a determinant of the route of the hysterectomy, even though multiple indications were present among some women. All analyses were performed using SAS version 9.3 software (SAS Institute Inc., Cary, North Carolina) and 2 -sided $p$ values $<0.05$ were considered significant.

Ethics approval for this study was granted by the University of British Columbia Research Ethics Board.

\section{Results}

\section{Study sample}

Between April 2007 and December 2011, 4372 women had an elective hysterectomy for a benign gynecologic indication in the Vancouver Coastal Health and Providence Health Care regions. Overall, 52.3\% of hysterectomies were abdominal, $25.5 \%$ were vaginal and $22.3 \%$ were laparoscopic. Taking vaginal and laparoscopic routes together, the overall rate of minimally invasive hysterectomies was $47.7 \%$. Over the study period, the proportion of hysterectomies performed abdominally decreased from $58.3 \%$ to $47.7 \%$, the proportion of hysterectomies performed vaginally decreased from $27.5 \%$ to $21.1 \%$, whereas the proportion of hysterectomies performed laparoscopically increased from $14.2 \%$ to $31.2 \%$. Overall, the rate of minimally invasive hysterectomies (vaginal or laparoscopic) increased from $41.7 \%$ to $52.3 \%$ ( $p<0.001$ for all trends; Figure 1).

\section{Unadjusted results}

Most of the participants (71.0\%) were between 40 and 60 years old. Uterine fibroids were the most common indication for hysterectomy $(52.1 \%)$, followed by menstrual bleeding disorder $(30.3 \%)$, prolapse $(22.0 \%)$, endometriosis $(21.5 \%)$ and pelvic pain $(6.7 \%)$. Most of the participants resided in urban areas $(94.0 \%)$, and most of the hysterectomies were performed in urban settings (95.0\%). Women who had a laparoscopic hysterectomy were younger on average, whereas most of the women who underwent vaginal hysterectomy were older on average. Rates of rural residence were higher women who had minimally invasive hysterectomies, whereas women with a lower socioeconomic status were more likely to have had an abdominal hysterectomy. Fibroids were associated with abdominal hysterectomy, whereas prolapse was associated with a vaginal approach. Similarly, a concurrent procedure for prolapse was more likely to occur with a vaginal approach to hysterectomy. Patient and hospital factors by route of hysterectomy are shown in Table 1.

\section{Adjusted results}

After adjusting for patient and hospital characteristics, the odds of using a minimally invasive approach to hysterectomy increased significantly between 2007 and 2011 (25\% increase in odds per year, 95\% CI 19\%-32\%; Table 2). The separate odds of either a laparoscopic or a vaginal approach relative to an abdominal approach also increased in recent years (Table 2).

\section{Factors for laparoscopic versus abdominal}

Patient factors associated with laparoscopic versus abdominal hysterectomy: the adjusted OR was higher for young age $(\leq 29 \mathrm{yrs})$ as a factor for choosing laparoscopic hysterectomy

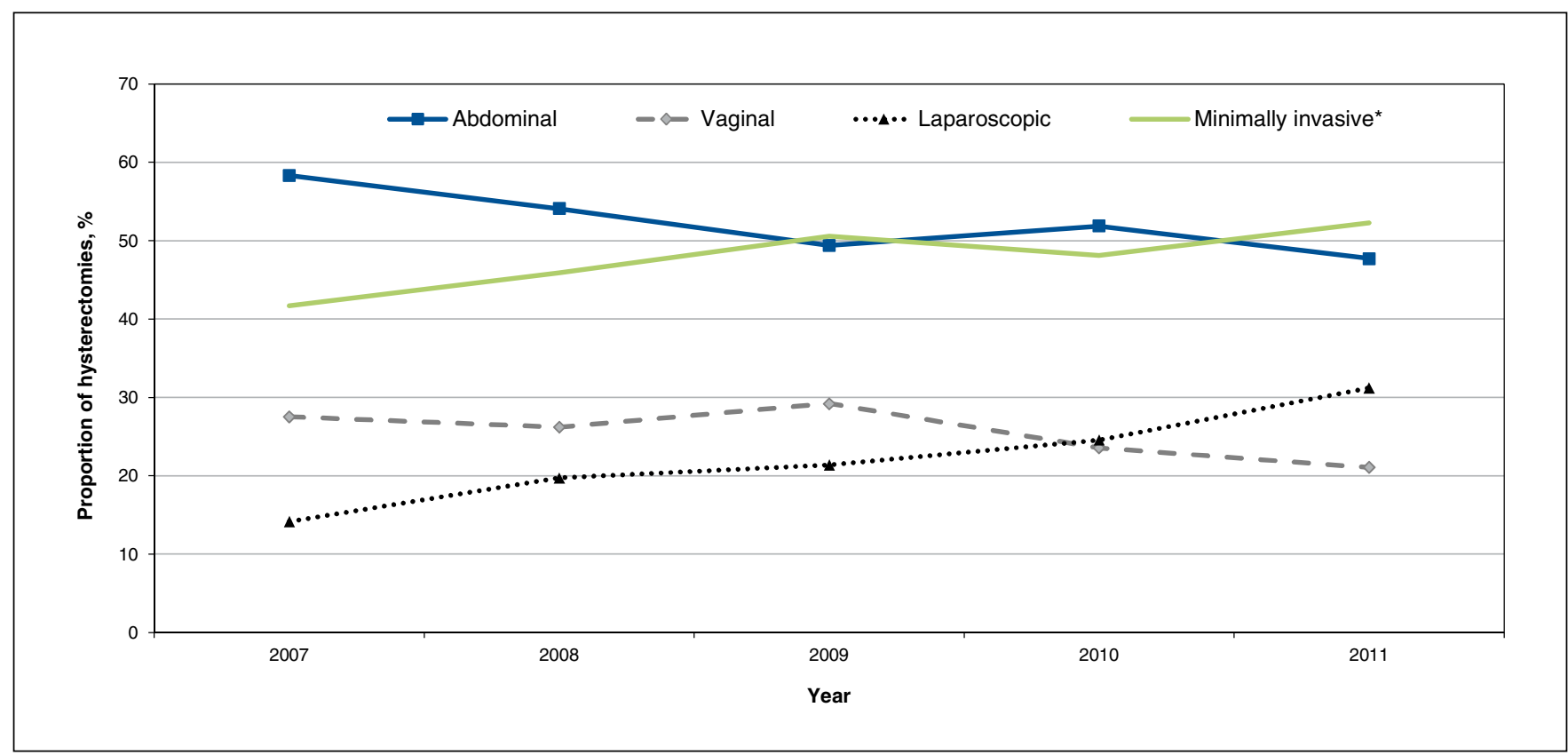

Figure 1: Temporal trends in elective abdominal, vaginal, laparoscopic and minimally invasive hysterectomies in women with benign indications, Vancouver Coastal Health and Providence Health Care regions, British Columbia, 2007-2011. *Minimally invasive hysterectomies include vaginal and laparoscopic hysterectomies. 


\section{OPEN}

Research

over the abdominal route (adjusted OR 4.59, 95\% CI 2.10 10.0 for $<30$ v. 40-49 yr), for rural residence (adjusted OR $1.89,95 \%$ CI 1.28-2.78), for pain as the main indication for hysterectomy (adjusted OR 2.08, 95\% CI 1.53-2.83) and for prolapse as the main indication of hysterectomy (adjusted OR 3.28, 95\% CI 2.14-5.03). Fibroids (adjusted OR 0.37,
95\% CI 0.29-0.46), a concurrent ovarian and prolapse procedure (adjusted OR 0.71, 95\% CI 0.58-0.86; and adjusted OR $0.50,95 \%$ CI $0.29-0.85$ ), and lower socioeconomic status (adjusted OR 0.58, 95\% CI 0.46-0.74 for lowest v. highest quintile) were associated with lower odds of using laparoscopic versus abdominal surgery. After adjusting for patient

Table 1: Patient and hospital characteristics associated with hysterectomy approach in women with benign indications (Vancouver Coastal Health and Providence Health Care regions, British Columbia, 2007-2011)

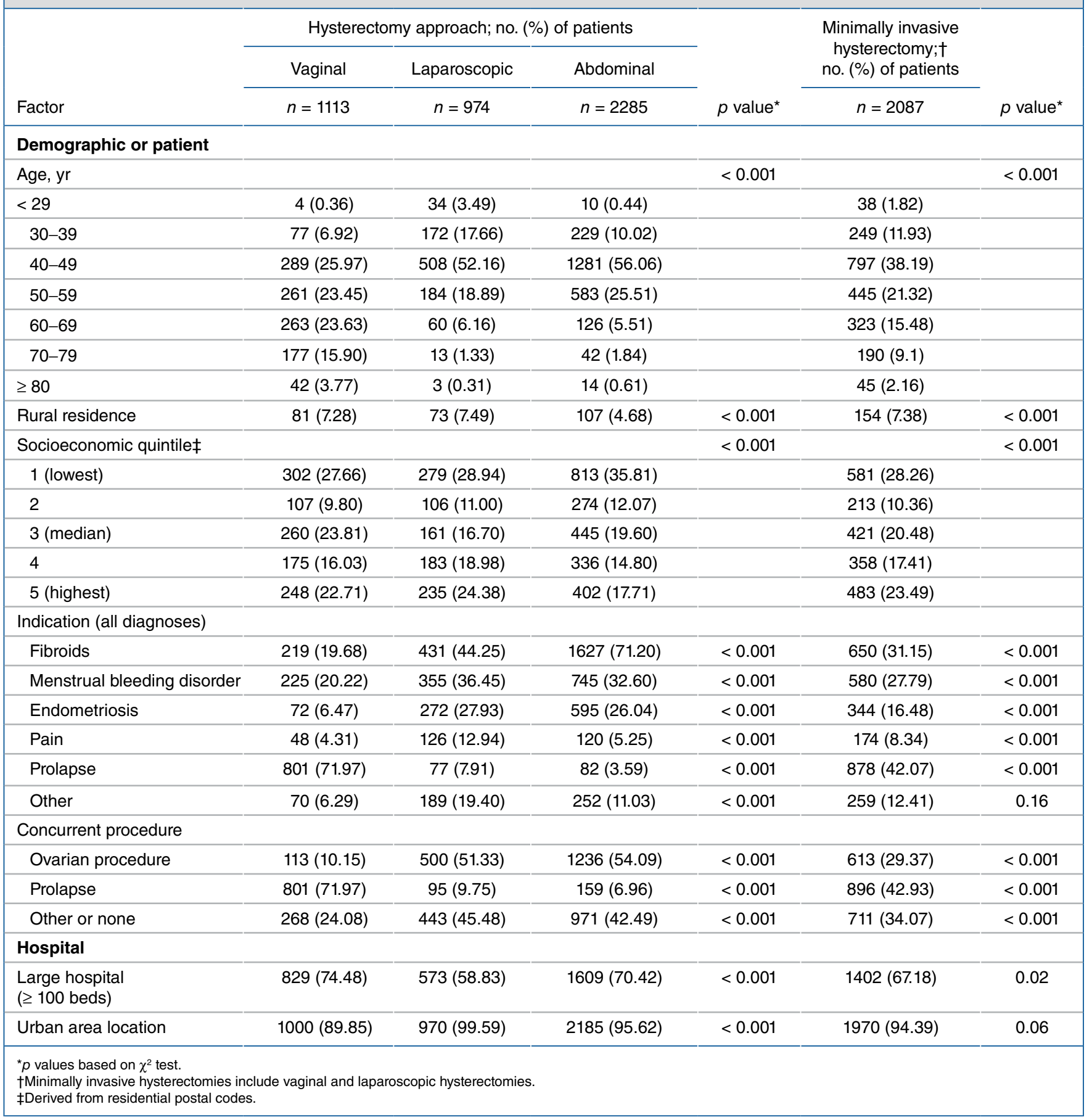


Table 2: Unadjusted and adjusted odds ratios for factors associated with elective vaginal, laparoscopic and minimally invasive hysterectomy among women with benign indications (Vancouver Coastal Health and Providence Health Care regions, British Columbia, 2007-2011)

\begin{tabular}{|c|c|c|c|c|c|c|}
\hline \multirow[b]{3}{*}{ Factor } & \multicolumn{6}{|c|}{ Hysterectomy } \\
\hline & \multicolumn{2}{|c|}{ Vaginal* $^{*}$} & \multicolumn{2}{|c|}{ Laparoscopic* } & \multicolumn{2}{|c|}{ Minimally invasive* } \\
\hline & OR $(95 \% \mathrm{Cl})$ & $\begin{array}{l}\text { Adjusted OR } \\
\qquad(95 \% \mathrm{Cl})\end{array}$ & OR $(95 \% \mathrm{Cl})$ & $\begin{array}{l}\text { Adjusted OR } \\
\qquad(95 \% \mathrm{Cl})\end{array}$ & OR $(95 \% \mathrm{Cl})$ & $\begin{array}{c}\text { Adjusted OR } \\
(95 \% \mathrm{Cl})\end{array}$ \\
\hline \multicolumn{7}{|c|}{ Demographic or patient } \\
\hline $\begin{array}{l}\text { Year of } \\
\text { procedure }\end{array}$ & $0.98(0.93-1.04)$ & $1.12(1.02-1.22)$ & $1.25(1.18-1.32)$ & $1.32(1.24-1.40)$ & $1.10(1.05-1.15)$ & $1.25(1.19-1.32)$ \\
\hline \multicolumn{7}{|l|}{ Age, yr } \\
\hline$\leq 29$ & $1.77(0.55-5.69)$ & $1.11(0.26-4.69)$ & $8.57(4.20-17.5)$ & $4.59(2.10-10.0)$ & $6.11(3.03-12.3)$ & $3.77(1.78-7.95)$ \\
\hline $30-39$ & $1.49(1.12-1.99)$ & $0.87(0.59-1.31)$ & $1.89(1.52-2.37)$ & $1.30(1.01-1.68)$ & $1.75(1.43-2.14)$ & $1.24(0.98-1.56)$ \\
\hline $40-49$ & Reference & Reference & Reference & Reference & Reference & Reference \\
\hline $50-59$ & $1.98(1.64-2.41)$ & $1.25(0.89-1.75)$ & $0.80(0.66-0.97)$ & $0.77(0.61-0.96)$ & $1.23(1.05-1.43)$ & $0.89(0.73-1.09)$ \\
\hline $60-69$ & $9.25(7.22-11.8)$ & $1.75(1.05-2.92)$ & $1.20(0.87-1.66)$ & $0.66(0.44-0.98)$ & $4.12(3.29-5.16)$ & $0.91(0.65-1.27)$ \\
\hline$\geq 70$ & $17.3(12.6-23.9)$ & $1.74(0.94-3.22)$ & $0.72(0.41-1.27)$ & $0.33(0.17-0.61)$ & $6.75(4.97-9.15)$ & $0.78(0.51-1.21)$ \\
\hline Rural residence & $1.60(1.19-2.15)$ & $1.12(0.63-2.01)$ & $1.65(1.21-2.24)$ & $1.89(1.28-2.78)$ & $1.62(1.26-2.09)$ & $1.62(1.14-2.29)$ \\
\hline \multicolumn{7}{|c|}{ Socioeconomic quintile $†$} \\
\hline 1 (lowest) & $0.58(0.47-0.71)$ & $0.81(0.56-1.16)$ & $0.58(0.47-0.72)$ & $0.58(0.46-0.74)$ & $0.58(0.49-0.69)$ & $0.62(0.50-0.77)$ \\
\hline 2 & $0.61(0.46-0.79)$ & $0.76(0.46-1.24)$ & $0.66(0.50-0.87)$ & $0.58(0.43-0.80)$ & $0.63(0.51-0.79)$ & $0.58(0.43-0.77)$ \\
\hline 3 (median) & $0.91(0.73-1.13)$ & $1.07(0.70-1.64)$ & $0.62(0.49-0.78)$ & $0.67(0.50-0.89)$ & $0.77(0.64-0.92)$ & $0.74(0.58-0.96)$ \\
\hline 4 & $0.81(0.64-1.03)$ & $0.87(0.58-1.29)$ & $0.93(0.73-1.18)$ & $0.86(0.66-1.11)$ & $0.86(0.71-1.05)$ & $0.86(0.68-1.09)$ \\
\hline 5 (highest) & Reference & Reference & Reference & Reference & Reference & Reference \\
\hline \multicolumn{7}{|c|}{ Indication (main diagnosis) } \\
\hline Fibroids & $0.23(0.18-0.3)$ & $0.24(0.18-0.33)$ & $0.37(0.3-0.45)$ & $0.37(0.29-0.46)$ & $0.31(0.26-0.37)$ & $0.31(0.26-0.38)$ \\
\hline $\begin{array}{l}\text { Menstrual } \\
\text { disorders }\end{array}$ & Reference & Reference & Reference & Reference & Reference & Reference \\
\hline Prolapse & $33.9(25.1-45.7)$ & $34.4(21.1-56.3)$ & $1.76(1.24-2.50)$ & $3.28(2.14-5.03)$ & $12.3(9.47-16.0)$ & $9.85(6.85-14.2)$ \\
\hline Other or none & $0.37(0.27-0.51)$ & $0.74(0.51-1.07)$ & $1.02(0.84-1.23)$ & $0.95(0.77-1.17)$ & $0.80(0.67-0.95)$ & $0.95(0.78-1.15)$ \\
\hline Pain & $1.70(1.12-2.57)$ & $1.53(0.95-2.46)$ & $2.20(1.66-2.91)$ & $2.08(1.53-2.83)$ & $2.02(1.55-2.63)$ & $1.89(1.42-2.52)$ \\
\hline Other & $0.66(0.46-0.96)$ & $1.81(1.14-2.89)$ & $1.11(0.84-1.46)$ & $1.37(1.00-1.87)$ & $0.92(0.72-1.18)$ & $1.34(1.02-1.78)$ \\
\hline \multicolumn{7}{|c|}{ Concurrent procedure } \\
\hline $\begin{array}{l}\text { Ovarian } \\
\text { procedure }\end{array}$ & $0.33(0.26-0.42)$ & $0.08(0.05-0.12)$ & $0.89(0.76-1.03)$ & $0.71(0.58-0.86)$ & $0.68(0.59-0.78)$ & $0.42(0.36-0.51)$ \\
\hline Prolapse & $34.0(26.0-44.5)$ & $1.68(1.07-2.64)$ & $0.87(0.57-1.34)$ & $0.50(0.29-0.85)$ & $13.4(10.4-17.2)$ & $2.28(1.59-3.26)$ \\
\hline Other or none & Reference & Reference & Reference & Reference & Reference & Reference \\
\hline \multicolumn{7}{|l|}{ Hospital } \\
\hline $\begin{array}{l}\text { Large hospital } \\
\text { ( } \geq 100 \text { beds) }\end{array}$ & $1.23(1.04-1.44)$ & $2.09(0.19-22.7)$ & $0.60(0.51-0.70)$ & $0.47(0.08-2.67)$ & $0.86(0.76-0.98)$ & $0.66(0.19-2.29)$ \\
\hline $\begin{array}{l}\text { Urban area } \\
\text { location }\end{array}$ & $0.41(0.54-0.31)$ & $0.25(0.02-3.18)$ & $11.1(30.3-4.07)$ & $22.2(2.6-192.3)$ & $0.77(0.59-1.01)$ & $1.84(0.47-7.32)$ \\
\hline $\begin{array}{l}\text { Note: Adjusted OR = } \\
\text { route. Adjusted for pe } \\
{ }^{*} \text { Minimally invasive } \mathrm{h} \\
\text { †Derived from reside }\end{array}$ & $\begin{array}{l}\text { adjusted odds ratio, Cl } \\
\text { riod (yr), age, rural res } \\
\text { ysterectomies include } \\
\text { ntial postal codes. }\end{array}$ & $\begin{array}{l}\text { onfidence interval, OR } \\
\text { ce, socioeconomic st } \\
\text { nal and laparoscopic }\end{array}$ & $\begin{array}{l}\text { dds ratio. Odds ratios } \\
\text { indication for surgery } \\
\text { rectomies. }\end{array}$ & $\begin{array}{l}\text { ese routes of hystere } \\
\text { urrent procedures an }\end{array}$ & $\begin{array}{l}\text { y are calculated relativ } \\
\text { spital characteristics. }\end{array}$ & o the abdominal \\
\hline
\end{tabular}


characteristics, urban hospital location was associated with using laparoscopic versus abdominal hysterectomy (adjusted OR 22.2, 95\% CI 2.6-192.3) (Table 2).

\section{Factors for vaginal versus abdominal}

Patient factors associated with undergoing vaginal hysterectomy include: the adjusted OR was higher for older age (adjusted OR 1.75, 95\% CI 1.05-2.92 for 60-69 v. 40-49 yr), for prolapse as the main indication (adjusted OR 34.4, 95\% CI 21.1-56.3) and for concurrent procedure for prolapse (adjusted OR 1.68, 95\% CI 1.07-2.64) (Table 2).

\section{Operative outcomes}

In terms of surgical outcomes, there was a significant difference in the median operative time for laparoscopic hysterectomies (median $140 \mathrm{~min}$, interquartile range 110-181 min) compared with abdominal (median $100 \mathrm{~min}$, interquartile range $78-132 \mathrm{~min}$ ) and vaginal (median $100 \mathrm{~min}$, interquartile range $76-130 \mathrm{~min})$ hysterectomies $(\mathrm{p}<0.0001)$. Length of hospital stay was also significantly different among the three routes (abdominal median $3 \mathrm{~d}$, interquartile range $2-3 \mathrm{~d}$; vaginal median $2 \mathrm{~d}$, interquartile range $1-3 \mathrm{~d}$; and laparoscopic median $1 \mathrm{~d}$, interquartile range, $1-2 \mathrm{~d} ; \mathrm{p}<0.0001$ ). No significant differences were observed for the prolonged hospital stay, return to the emergency department or readmission to hospital factors (Table 3 ).

\section{Discussion}

\section{Main findings}

The proportion of minimally invasive hysterectomies steadily increased from $41.7 \%$ to $52.3 \%$ between 2007 and 2011 in the Vancouver Coastal Health and Providence Health Care regions. Factors associated with the choice of laparoscopic over abdominal hysterectomy were surgery performed recently, younger age, pain or prolapse as the main indication, living at a rural residence and higher socioeconomic status. Compared with the abdominal route, the use of a laparoscopic route was less likely to happen in women with fibroids, those with either a concurrent prolapse or an ovarian procedure, and women with a lower socioeconomic status. Factors associated with the choice of vaginal over abdominal hysterectomy were surgery performed recently, older age and an indication of vaginal prolapse. Hospital characteristics were not significantly associated with a minimally invasive approach after adjustment for patient characteristics, except for the laparoscopic approach, which was more likely to be performed in urban area hospitals.

\section{Comparison with other studies}

The observed rate of minimally invasive hysterectomy is consistent with the $41 \%$ rate reported in Ontario in $2007,{ }^{11,12}$ and with the temporal rise from $39.9 \%$ in $2002-2003$ to $44.3 \%$ in 2008-2009 reported in Quebec. ${ }^{13}$ In a national survey of endoscopic practice, lack of training was identified as a major barrier to the use of laparoscopy for hysterectomy; therefore, the recent increase in minimally invasive hysterectomies may reflect the increased exposure to laparoscopic hysterectomy in residency and fellowship training programs. Furthermore, several mentorship programs were present in the Vancouver Coastal Health region, and our results may reflect the effectiveness of such initiatives.

We observed an overall decline in vaginal hysterectomies. One reason for the temporal decline in crude rates may be changes in patient characteristics over time. For example, it is possible that women with structurally normal uteri who would have been candidates for vaginal hysterectomy increasingly benefitted from effective, conservative treatments for menstrual bleeding disorders, such as hormonal or ablative treatments, and, therefore, they were less likely to require hysterectomy. This is consistent with the observation that fibroids represented the most common indication for hyster-

Table 3: Surgery outcomes associated with route of hysterectomy in women with benign indications (Vancouver Coastal Health and Providence Health Care regions, British Columbia, 2007-2011)

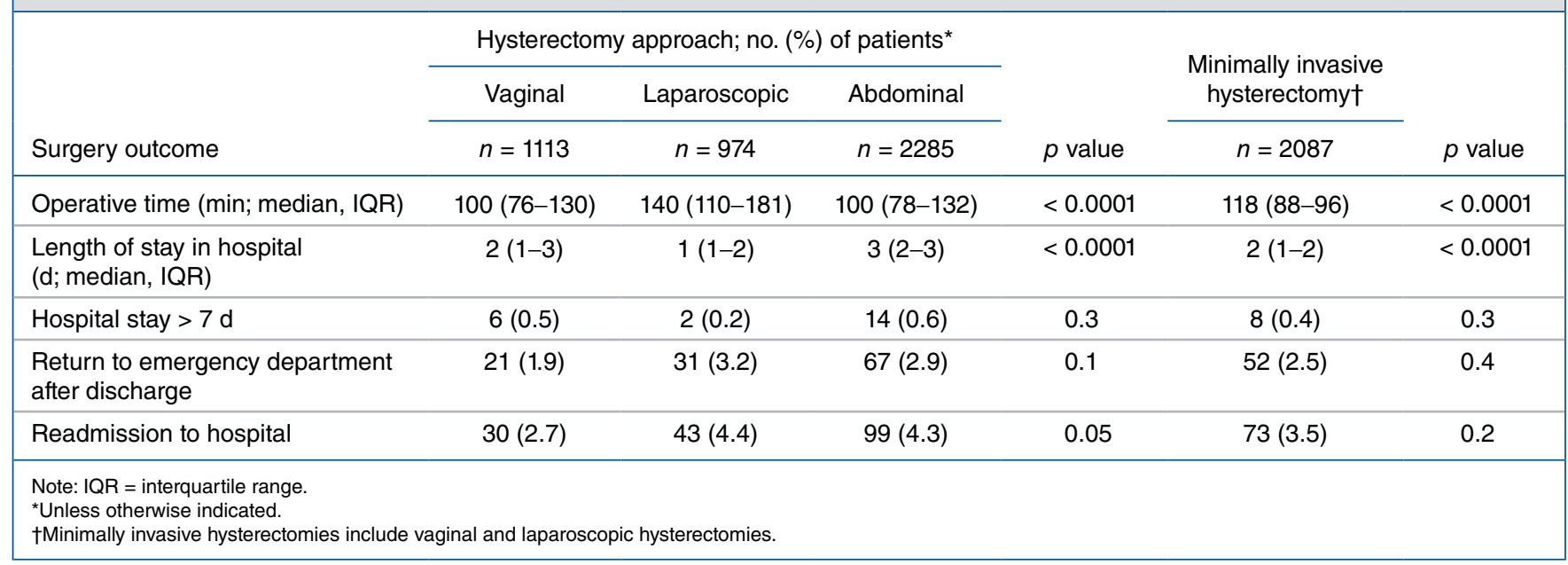


ectomy, and the multivariate analysis that showed that the odds of vaginal hysterectomy increased significantly over the study period by $12 \%$ per year (95\% CI 1\%-22\%) compared with abdominal hysterectomy when patient characteristics were controlled. Furthermore, prophylactic salpingectomy for the prevention of ovarian cancer has become more common in British Columbia in recent years. Because salpingectomy using the vaginal route can be technically challenging compared with the laparoscopic route, it is possible that more laparoscopic than vaginal hysterectomies have been performed for this purpose.

We also found the seemingly contradictory result that concurrent procedure was a factor associated with both abdominal (versus laparoscopic) and vaginal (versus abdominal) routes. Most women with prolapse underwent vaginal hysterectomy with a concurrent prolapse procedure; however, there was a group of women who had a concurrent prolapse procedure (e.g., hysterectomy and concurrent colposacropexy) but did not have vaginal surgery. In these patients, the hysterectomy and the concurrent procedure were performed using the abdominal route.

Whereas it is generally accepted that patient clinical factors will determine the route of hysterectomy, the observed association between social and demographic factors and route of hysterectomy was less expected. We observed that women with lower socioeconomic status were less likely to undergo laparoscopic hysterectomy, after adjustment for concurrent factors. It is possible that the relationship between route of hysterectomy and socioeconomic status could be confounded by patient comorbidities that were not documented in the data source; however, the association between routes of hysterectomy and socioeconomic status, race and geographic location has been reported previously. ${ }^{19-21}$ The route of hysterectomy varies by geographic region in Ontario, with some local health areas reporting a 2 -fold higher rate of laparoscopic hysterectomy compared with the rates of other routes (63\% v. 30\%). ${ }^{11,12}$ However, these studies showed that the route of hysterectomy did not vary significantly with neighbourhood income or neighbourhood educational attainment. ${ }^{11,12}$ Further population-based studies in Canada are needed to corroborate these findings.

\section{Strengths and limitations}

The use of a large and validated data source, such as Discharge Abstract Database, as the core population-based dataset (with multiple hospitals within a defined geographical area, urban and rural locations, and an adequate study size) is a major strength of this study. However, not all hospitals in the province of British Columbia were included, and thus our analysis may not be generalizable outside of the 2 health regions included in our analysis. Other limitations include the lack of detailed clinical information, such as patient body mass index and comorbid conditions. Furthermore, because route of hysterectomy is partially dependent on the ability and preference of the surgeon, the lack of information about the surgeon in our dataset is a limitation. In addition, we could not adequately identify the infrequent cases where laparoscopy was converted to laparotomy; however, because of the small number of cases, the effect on study outcome is expected to be very small. Finally, underreporting and coding errors inherent in the use of hospital administrative data may have been present in the dataset.

\section{Conclusion}

Approximately half of all hysterectomies in the Vancouver Coastal Health and Providence Health Care regions are performed using a minimally invasively approach; the frequency of use of this type of approach has increased substantially in recent years. Vaginal hysterectomies are associated with patient clinical factors, whereas laparoscopic hysterectomies are associated with clinical, sociodemographic and hospital characteristics. The association between route of hysterectomy and socioeconomic status in a Canadian population observed in this study is noteworthy and warrants corroboration with future studies.

\section{References}

1. Millar WJ. Hysterectomy, 1981/82 to 1996/97. Health Rep 2001;12:9-22.

2. Laberge PY, Singh SS. Surgical approach to hysterectomy: introducing the concept of technicity. 7 Obstet Gynaecol Can 2009;31:1050-3.

3. Nieboer TE, Johnson N, Lethaby A, et al. Surgical approach to hysterectomy for benign gynaecological disease. Cochrane Database Syst Rev 2009;CD003677.

4. Garry R, Fountain J, Mason S, et al. The eVALuate study: two parallel randomised trials, one comparing laparoscopic with abdominal hysterectomy, the other comparing laparoscopic with vaginal hysterectomy [published erratum in BMJ 2004;328:494]. BM7 2004;328:129.

5. Roy KK, Goyal M, Singla S, et al. A prospective randomised study of total laparoscopic hysterectomy, laparoscopically assisted vaginal hysterectomy and non-descent vaginal hysterectomy for the treatment of benign diseases of the uterus. Arch Gynecol Obstet 2011;284:907-12.

6. ACOG committee opinion no. 444: Choosing the route of hysterectomy for benign disease. Obstet Gynecol 2009;114:1156-8.

7. Lefebvre G, Allaire C, Jeffrey J, et al. SOGC clinical guidelines. Hysterectomy. 7 Obstet Gynaecol Can 2002;24:37-61.

8. McCracken G, Lefebvre GG. Vaginal hysterectomy: dispelling the myths. 7 Obstet Gynaecol Can 2007;29:424-8.

9. Al-Khaduri M, Al-Farsi Y. Technicity as a quality indicator of excellence in gynaecology. Sultan Qaboos Univ Med F 2012;12:93-6.

10. Chen I, Bajzak KI, Guo Y, et al. A national survey of endoscopic practice among gynaecologists in Canada. 7 Obstet Gynaecol Can 2012;34:257-63.

11. Dunn S, Wise MR, Johnson LM, et al. Reproductive and gynaecological bealth. In Bierman AS, editor. Project for an Ontario women's health evidence-based report: volume 2. Toronto: Institute for Clinical Evaluative Sciences; 2012. Available: http://powerstudy.ca/power-report/volume2/reproductive-gynaecological-health/.

12. Bierman AS, ed. Project for an Ontario women's health evidence-based report: volume 2. Toronto: St. Michael's Hospital; Institute for Clinical Evaluative Sciences; 2012. Available: http://powerstudy.ca/.

13. Bernatchez-Laflamme SM, Bujold E, Roberge S, et al. Évolution de l'indice de technicité des hystérectomies au Québec. 7 Obstet Gynaecol Can 2013;35:144-8.

14. Data quality documentation, discharge abstract database - Multi-year information. Ottawa: Canadian Institute for Health Information; 2012.

15. Martin JB, Cantrell ME, Fichman RG, et al. An integrated surgical suite management information system. 7 Med Syst 1984;8:287-99.

16. Chu T, Demlow E, Fontaine J, et al.; Public Health Surveillance Unit. An investment in regional public bealth, highlights from the first five years, 2007-2012. Vancouver: Vancouver Coastal Health; 2013.

17. Postal code conversion file (PDDF), reference guide. Ottawa: Statistics Canada; 2004. Cat no 92F0153GIE. Available: http://dsppsd.pwgsc.gc.ca/Collection/Statcan /92F0153GIE/92F0153GIE2005001.pdf. (accessed 2014 Aug. 26).

18. Mustard CA, Derksen S, Berthelot JM, et al. Assessing ecologic proxies for household income: A comparison of household and neighbourhood level income measures in the study of population health status. Health Place 1999;5:157-71.

19. Jacoby VL, Autry A, Jacobson G, et al. Nationwide use of laparoscopic hysterectomy compared with abdominal and vaginal approaches. Obstet Gynecol 2009;114:1041-8.

20. Abenhaim HA, Azziz R, Hu J, et al. Socioeconomic and racial predictors of undergoing laparoscopic hysterectomy for selected benign diseases: analysis of 341487 hysterectomies. 7 Minim Invasive Gynecol 2008;15:11-5.

21. Health indicators 2010. Ottawa: Canadian Institute for Health Information; 2010. Available: https://secure.cihi.ca/free_products/Healthindicators2010_en.pdf (accessed 2014 Aug. 26). 
Affiliations: Department of Obstetrics and Gynaecology (Chen), University of Ottawa, Ottawa, Ont.; School of Population and Public Health (Chen, Joseph) University of British Columbia, Vancouver; Department of Obstetrics and Gynaecology (Lisonkova, Allaire, Williams, Yong, Joseph), University of British Columbia, Vancouver, BC

Contributors: All of the authors contributed equally to the design and conduct of the study, revised the manuscript, approved the final version submitted for publication and agreed to act as guarantors of the work.

Funding: Innie Chen received a Frederick Banting and Charles Best Canada Graduate Scholarship Award from the Canadian Institutes of
Health Research. K.S. Joseph is supported by a Chair in Maternal, Fetal and Infant Health Services Research from the Canadian Institutes of Health Research. This study was funded by a grant from the Canadian Institutes of Health Research (APR-126338).

Acknowledgements: We thank the Vancouver Coastal Health Authority Decision Support unit and Providence Health Care Decision Support unit for providing the data used in this study.

Supplemental information: For reviewer comments and the original submission of this manuscript, please see www.cmajopen.ca/content/2/4 /E273/suppl/DC1 